\title{
Simulation methods for complicated polymer networks
}

\begin{abstract}
Molecular simulations have received extensive applications in various fields with well-defined molecular structures. The simulations of network polymers are still limited because of their irregular and complicated structures. This mini review intends to provide a summary of the efforts made for the simulations of network polymers, and an effective self-assembling method is suggested for the generation of random polymer networks.
\end{abstract}

Keywords: molecular simulations, telechelic chains, random branching, synthetic polymers, molecular dynamics, polymer network, morse potential, WCA potential, random collision, network defects
Volume I Issue 4 - 2017

\author{
Rongliang Wu \\ College of Material Science and Engineering, Donghua \\ University, China
}

\begin{abstract}
Correspondence: Rongliang Wu, College of Material Science and Engineering, State Key Laboratory for Modification of Chemical Fibers and Polymer Materials, Donghua University, Shanghai 201620, China, Tel +862I 67792914 , Email wurl@dhu.edu.cn
\end{abstract}

Received: April 30, 2017| Published: August 14, 2017

\section{Introduction}

Molecular simulations have boomed in recent years for natural macromolecules such as proteins, nucleic acids and polysaccharides. Different sets of molecular force fields, such as CHARMM, AMBER, OPLS and GROMOS etc. have been developed for such bio-related systems aiming at accurate prediction of molecular motions in the microscopic world. Albeit great progress in these natural systems, the simulations of synthetic polymers are fairly limited. Up to now, even a general force field describing most synthetic polymers is scarcely seen because of their greater varieties of the repeat units. Worse still, the most important category of synthetic polymers has network structure with random cross-links between chains of different length. Such complicated network polymers are clearly beyond the capability of classical quantum chemistry or the reactive force field methods. Normal simulations can only be limited to polymers with well-defined structures, and new methods for the generation of random network polymer structures are necessary.

To mimic the polymer network systems in greatest extent, defect-free diamond-like network topologies with connections to the periodic images were generated, ${ }^{1,2}$ the influence of defects were also investigated with varying cross-linking densities. ${ }^{3}$ More realistic network structures with random cross-links were also tried with the probabilistic algorithms, in which the molecules were essentially static and the capture radius for the cross-linking reaction was gradually incremented. ${ }^{4}$ Dynamic processes of the bond fluctuation model in Monte Carlo simulations were also used for the reactive cross linking of polymer chains, ${ }^{5-9}$ while it did not give realistic kinetics of the whole systems.

Molecular dynamics (MD) simulation is a proper choice for such dynamic processes. Meanwhile, non-natural arbitrary distance cutoffs from $0.4-1.0 \mathrm{~nm}$ were often used for the preparation of networks of bead-spring homopolymers with poly-functional cross-linkers at chain ends. ${ }^{10}$ Similar methods were also used for more realistic models of cross-linked poly (methacrylates). ${ }^{11}$ Different assumptions of the amine reactivity and connection procedures were also used to build epoxy-based polymer networks. ${ }^{12,13}$ The dynamic cross-linking approach, whose potential reactive pairs were reacted simultaneously instead of connecting only the nearest pair at a time, was found to be more realistic in building large polymer networks with thousands of atoms. ${ }^{14}$ In addition to the deficiencies of conversion rates at the late stage and the too-large cut-offs implemented,$^{14}$ the manual introduction of emergent bonds to the MD algorithm might make the whole process non-consecutive in binary. Self-association of telechelic polymer chains with screened attractive Coulomb potential, ${ }^{15,16}$ multi-ranged potentials ${ }^{17}$ or even the Lennard-Jones potential ${ }^{18}$ were also used to model the formation of gel polymers. Meanwhile, the functionalities of the associative ends could not be well monitored, and micelle-like aggregations with uncontrollable number of chains ends were obtained. ${ }^{16,18}$

Based upon the united-atom polyethylene force field, ${ }^{19}$ a realistic model of random polymer networks was developed with telechelic chains, whose maximal functionality of the cross-linker could be easily adjusted by the relative size of the particles. ${ }^{20}$ The shifted Morse potential at the inter-atomic distance $r, V_{\text {morse }}(r)$, was introduced to normal molecular dynamics simulations to govern the cross-linking of polymer chains with special end groups.

$V_{\text {morse }}(r)=D\left[1-e^{-\beta(r-b)}\right]^{2}-D=D\left(e^{2 \beta(b-r)}-2 e^{\beta(b-r)}\right)$

In which $D$ is the reactive potential well depth, $b$ is the reactive bond length and $\beta$ governs the reactive interaction width. It was demonstrated that the bonding of the reactive particles abided by the dynamic cross-linking process, as illustrated in Figure 1A. All particles had the same reactivity and the random collisions between such reactive particles lead to increasing fractions of central particles with 1-4 end group particles. Because of the short-ranged nature of the reactions, the reactive potential introduced had almost no interference to the population of other particles in the systems. ${ }^{20}$ One of the random polymer networks formed through chain-end cross-linking with such reactive particles at both ends is shown in Figure 1B, in which the functionality of the cross-linker was also set to 4 . The conversion 
of polymer chains into the large polymer network was efficient seeing from the fractions of end-groups or chains reacted in Table 1. Furthermore, setting the maximum functionality was done through the adjustment of end-group collision size, and the same method could also be used for linear polymerization of small molecules when setting the maximum functionality to 2 , or the initiation of polymer branching after setting to $3 .^{21}$ Thus, the self-assembling method of controllable reaction of linear chains made it possible for exact molecular simulation of multi-disperse molecular weight polymers, branched polymers as well as complicated polymer networks, in which even defects were statistically tractable. ${ }^{22}$

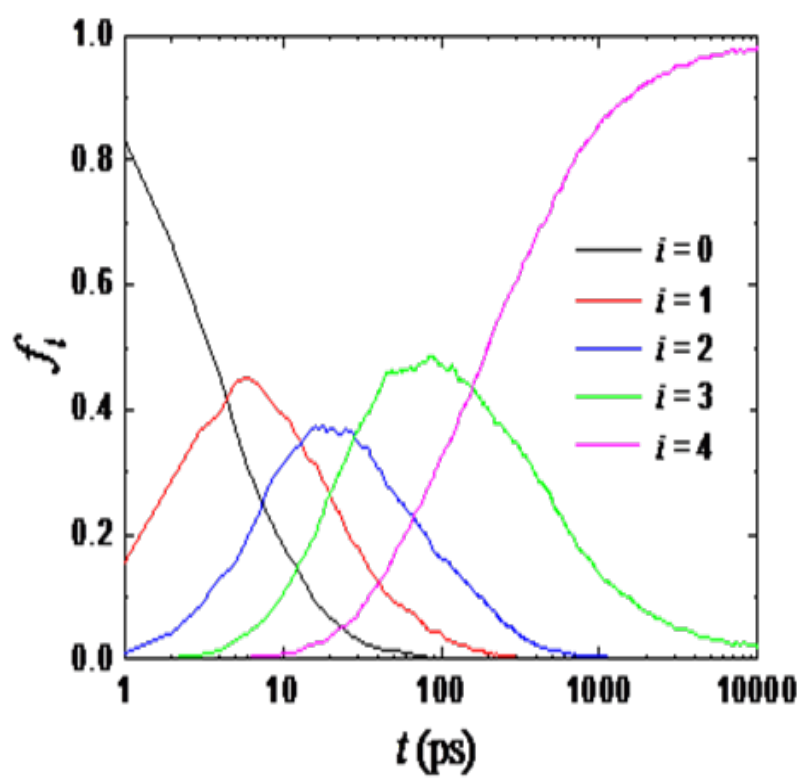

Figure I(A) The association process of particles with maximum functionality of 4 with the evolution of simulation time $t$, in which $f_{i}$ is the fraction of reacted central particles with $\mathrm{i}$ end group particles.

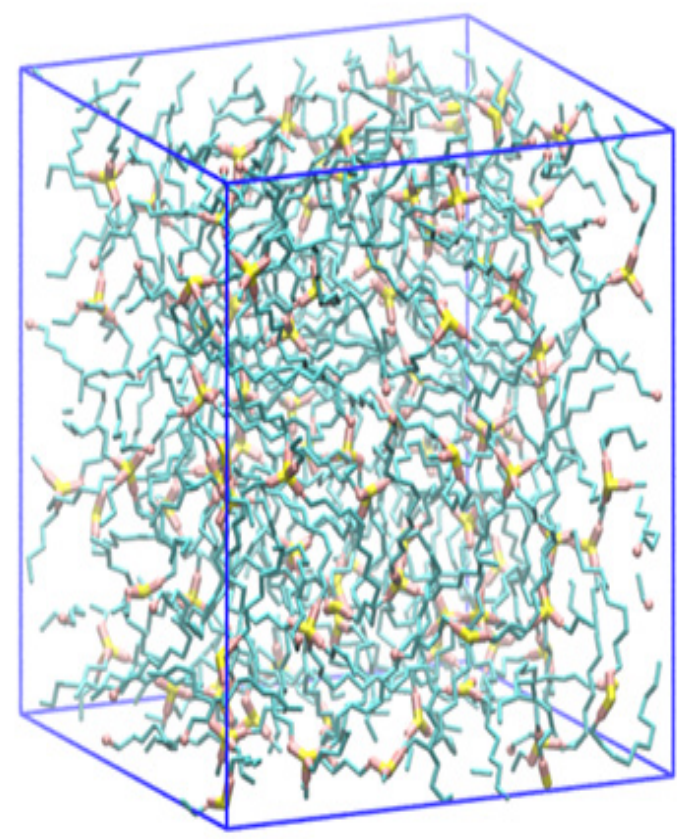

Figure I(B) The network polymer formed through random cross-linking.
Table I The properties of polymer networks obtained with different chain lengths

\begin{tabular}{lllllll}
\hline $\mathbf{N}$ & $\mathbf{f}_{\mathrm{i}=4}$ & $\mathbf{f}_{\mathrm{E}}$ & $\mathbf{f}_{\text {chain }}$ & $\mathbf{f}_{\text {av }}$ & $\mathbf{N}_{\text {dangleE }}$ & $\mathbf{N}_{\text {loop }}$ \\
\hline 10 & $4(76 \%)$ & $94.00 \%$ & $100 \%$ & 3.76 & 24 & 0 \\
14 & $4(73 \%)$ & $93.00 \%$ & $99.00 \%$ & 3.71 & 28 & 3 \\
20 & $4(68 \%)$ & $90.00 \%$ & $98.50 \%$ & 3.61 & 40 & $\mathrm{I}$ \\
30 & $4(73 \%)$ & $93.00 \%$ & $98.50 \%$ & 3.73 & 28 & 3 \\
50 & $4(90 \%)$ & $97.50 \%$ & $100 \%$ & 3.9 & 10 & 2 \\
100 & $4(84 \%)$ & $96.00 \%$ & $100 \%$ & 3.84 & 16 & 5 \\
\hline
\end{tabular}

$f_{i}=4$ : Fractions of cross-linkers reacted.

$f_{E}$ : Fractions of end groups reacted.

$\mathrm{F}_{\text {chain }}:$ Fractions of chains reacted.

$\mathrm{F}_{\mathrm{av}}$ :Average functionality of the cross-linkers.

$\mathrm{N}_{\text {dangle: }}$ : Number of dangling ends.

$\mathrm{N}_{\text {loop: }}$ : Number of loops.

\section{Acknowledgements}

This work was supported by the National Science Foundation of China (No. 21304017 and 21471030), the Fundamental Research Funds for the Central Universities, and the Innovation Program of Shanghai Municipal Education Commission, and the Innovation Program of Shanghai Science and Technology Commission (No. 14521100600). We acknowledge the provision of computational resources from the supercomputing center of the Chinese Academy of Sciences.

\section{Conflict of interest}

The author declares no conflict of interest.

\section{References}

1. Everaers R. Entanglement effects in defect-free model polymer networks. New J Phys. 1999;1(12):1-55.

2. Schneider S, Linse P. Monte carlo simulation of defect-free cross-linked polyelectrolyte gels. J Phys Chem B. 2003;107(32):8030-8040.

3. Edgecombe S, Linse P. Monte carlo simulation of polyelectrolyte gels: effects of polydispersity and topological defects. Macromolecules. 2007;40(10):3868-3875.

4. Escobedo FA, Pablo JJd. Molecular simulation of polymeric networks and gels: phase behavior and swelling. Physics Reports. 1999;318(3):85112 .

5. Saalwächter K, Kleinschmidt F, Sommer JU. Swelling heterogeneities in end-linked model networks: a combined proton multiple-quantum nmr and computer simulation study. Macromolecules. 2004;37(23):85568568 .

6. Aguilera-Mercado BM, Genesky GD, Duncan TM, et al. ${ }^{2} \mathrm{H}$ NMR and simulation studies of chain segment orientation in PDMS bimodal networks. Macromolecules. 2010;43(17):7173-7184.

7. Balabanyan AG, Kramarenko EY, Ronova IA, et al. Monte Carlo study of structure and kinetics of formation of end-linked polymer networks. Polymer. 2005;46:4248-4257. 
8. Chen YC, Chiu WY. Polymer Chain Bulldup and Network Formation of Imidazole-Cured Epoxy/Phenol Resins. Macromolecules. 2000;33(18):6672-6684.

9. Wang S, Chen CC, Dormidontova EE. Reversible association and network formation in 3:1 ligand-metal polymer solutions. Soft Matter. 2008;4(10):2039-2053.

10. Gresta GS, Kremerb K, Duering ER. Kinetics and relaxation of end crosslinked polymer networks. Physica A: Statistical Mechanics and its Applications. 1993;194(1):330-337.

11. Doherty DC, Holmes BN, Leung P, et al. Polymerization molecular dynamics simulations. I. Cross-linked atomistic models for poly (methacrylate) networks. Comp and Theor Polymer Science. 1998;8:169-178.

12. $\mathrm{Wu} \mathrm{C}, \mathrm{Xu} \mathrm{W}$. Atomistic molecular modelling of crosslinked epoxy resin. Polymer. 2006;47:6004-6009.

13. Yarovsky I, Evans E. Computer simulation of structure and properties of crosslinked polymers: application to epoxy resins. Polymer. 2002;43:963-969.

14. Varshney V, Patnaik SS, Roy AK, et al. A Molecular dynamics study of epoxy-based networks: cross-linking procedure and prediction of molecular and material properties. Macromolecules. 2008;41(18):6837-6842.

15. Khalatur PG, Khokhlov AR, Kovalenko JN, et al. Molecular dynamics study of the solution of semiflexible telechelic polymer chains with strongly associating end-groups. J Chem Phys. 1999;110(12):6039-6049.
16. Ayyagari C, Bedrov D, Smith GD. Equilibrium sampling of self-associating polymer solutions: A parallel selective tempering approach. $J$ Chem Phys. 2005;123(12):124912.

17. Heine DR, Grest GS, Lorenz CD, et al. Atomistic simulations of end-linked poly(dimethylsiloxane) networks: structure and relaxation. $\mathrm{Ma}$ cromolecules. 2004;37(10):3857-3864.

18. Cruz MOdl, Ermoshkin AV, Carignano MA, et al. Analytical theory and Monte Carlo simulations of gel formation of charged chains. Soft Matter. 2009;5(3):629-636.

19. Paul W, Yoon DY, Smith GD. An optimized united atom model for simulations of polymethylene melts. J Chem Phys. 1995;103(4):1702-1709.

20. Wu RL, Li T, Nies E. Langevin dynamics simulation of chain crosslinking into polymer networks. Macromol Theor Simul. 2012;21(4):250 265 .

21. Fu K, Liu J, Qiu X, et al. The molecular simulation method for functionality controllable polymerization. Computers and Applied Chemistry. 2016;33:898-904

22. Wu RL, Li T, Nies E. Polymer networks by molecular dynamics simulation: Formation, thermal, structural and mechanical properties. Chinese J Polym Sci. 2013;31(1):21-38. 